\title{
Generating Reduced Order Models using Subspace Iteration for Linear RLC Circuits in Nanometer Designs
}

\author{
J.V.R.Ravindra, M.B.Srinivas \\ Center for VLSI and Embedded System Technologies (CVEST), \\ International Institute of Information Technology, \\ Gachibowli, Hyderabad 500 032, India \\ ravindra@research.iiit.ac.in, srinivas@iiit.ac.in
}

\begin{abstract}
This paper presents a model order reduction technique using subspace iterative scheme for high speed coupled integrated circuit interconnects in nanometer designs. The salient feature of this technique is less complexity in computation of a few smallest poles of the reduced order model. This paper shows that the subspace iterative scheme produces reduced systems that accurately follow the time- and frequency- domain responses of the original system. Experimental results show that the subspace iterative scheme achieves more accuracy than the variational Krylov-subspace-based model order reduction techniques. Significant reduction in computational expense is achieved as the size of the reduced equations is much less than that of the original system.
\end{abstract}

\section{Categories and Subject Descriptors}

B.7.1 [Types and Design Styles]: VLSI (very large scale integration)

\section{General Terms \\ Design}

\section{Keywords}

Model Order Reduction, Interconnects, Krylov-subspace

\section{INTRODUCTION}

The recent trend in the VLSI industry toward nanometer designs, low power consumption, high speed digital circuits and increased integration of analog circuits with digital blocks has made the signal integrity analysis a challenging task. When analyzing high-performance integrated circuit designs, it is well known that the single lumped resistor- capacitor (RC) model of interconnect is insufficiently accurate. It has been shown [1] that reasonably accurate electro-quasistatic, or transient interconnect, simulations could be performed by computing the time evolution of the electric field both inside and outside the conductors via a finite-difference discretization of Laplace's equation. A boundary-element approach

Nano-Net 2007 September 24-26, 2007, Catania, Italy.

Copyright 2007 ICST ISBN 978-963-9799-10-3

DOI 10.4108/ICST.NANONET2007.2084
[2] based on Green's theorem was proposed which performs the calculation using the same surface discretization used for ordinary capacitance extraction, thereby avoiding the large exterior domain mesh and computation. To verify the effects induced by interconnects a combination of extraction and analysis is necessary. Extraction determines the resistance, the capacitance and the inductance of interconnects, which can then be used to build a circuit model for the analysis of interconnect effects. For analysis (or simulation), research has been carried out of the use of model order reduction (MOR) [3-5], complex frequency hopping[6-8], Krylov-space techniques $[9,10]$ and multi conductor transmission lines [1113]. Model order reduction is based on approximating the Laplace-domain transfer function of a linear (or linearized) network by a relatively small number of dominant poles and zeros. Such reduced order models can be used to predict the time-domain or frequency-domain response of the linear network. A multipoint moment-matching or complex frequency hopping $(\mathrm{CFH})$ technique extracts accurate dominant poles of a linear sub-network up to any predefined maximum frequency. This method generates a single transfer function for a large linear sub-network and provides for a CPU/accuracy tradeoff. Model order reduction can also be obtained using projecting the original system described by nonlinear differential equations into Krylov subspace of lower dimension. This reduced model can be simulated using conventional numerical techniques. Simulation of interconnects can also be performed treating the interconnect as full-wave model. But a few researchers have attempted to address the problem of process aware model order reduction. A multivariate moment matching technique is used in [14], where parameters are assumed to be linearly separable. A variational analysis approach is taken in [15] for resistor-capacitor-inductor (RCL) interconnect modeling with statistically varying parameters. An interpolation technique is proposed in [16] for parametric interconnect analysis. SBT technique [17] is proposed for tightly coupled interconnects with process variations. All these methods pose specific assumptions on the models, and their potential for general applications is limited. In this paper, to start with, the telegrapher's equations have been considered to represent the large VLSI circuit to reduce the order using subspace iterative scheme [18].

This paper is organized as follows: Section 2 gives an overview of the model order reduction. Section 3 provides a mathematical framework of dynamic RLC circuits. The proposed algorithm is explained in Section 4. Section 5 discusses the simulation results including the comparison between the pro- 
posed model reduction technique and different model-order reduction techniques in [3-5]. Finally, Section 6 presents the concluding remarks for this paper.

\section{MODEL ORDER REDUCTION (MOR)}

Model order reduction is a technique that takes a circuit containing a large number of poles and reduces it to a smaller representation consisting of the dominant poles from the original linear circuit as shown in Fig. 1. There are two approaches to model order reduction: moment matching [1416] and matrix approximation [19].

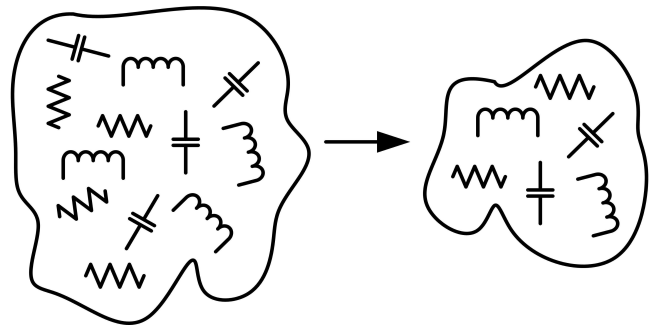

Figure 1: Model Order Reduction

A lumped linear time-invariant circuit can be described by first-order differential equations

$$
\begin{aligned}
& \dot{x}=A x+B u \\
& y=C x+D u
\end{aligned}
$$

where $u \in \Im^{m}$ is the external stimulus to the system, $B \in$ $\Im^{n \times m}$ is the input matrix, $x \in \Im^{n}$ is the state vector, $C \in$ $\Im^{n \times m}$ is the output matrix, $y \in \Im^{p}$ is the output of the model, $D \in \Im^{p \times n}$ denotes the direct coupling term and is the transformation matrix. We wish to obtain the zerostate impulse response of a linear circuit described by (1), which in turn can be used to determine its response to any excitation. We apply the Laplace transform to (1) assuming zero initial conditions and ignoring the term, which can be treated separately. Then, we obtain

$$
\begin{aligned}
s X & =A X+B U \\
Y & =C X
\end{aligned}
$$

Where $X, Y$, and $U$ denote the Laplace transform of $x, y$, and $u$ respectively. It follows from eq. (2) that the transfer function, or the Laplace transform of the impulse response, defined as $\mathrm{H}(\mathrm{s})=\mathrm{Y}(\mathrm{s}) / \mathrm{U}(\mathrm{s})$, is given by

$$
H(s)=C(s I-A)^{-1} B
$$

Where $I$ is identity matrix. $H(s)$ can be expanded using Maclaurin series

$$
H(s)=\sum_{i=0}^{\infty} m_{i} s^{i}
$$

Comparing eq (3) and (4) and equating like powers of $s$, it can be shown that

$$
m_{i}=-C A^{-i-1} B, \quad i=0,1,2 \cdots \cdots
$$

In a reduced-order model, especially one obtained by moment matching, the transfer function is approximated by the reduced-order system of proper rational function of ' $s$ ' having $q$-poles

$$
H(s)=\frac{n_{q-1} s^{q-1}+n_{q-2} s^{q-2}+\cdots \cdots+n_{1} s+n_{0}}{s^{q}+d_{q-1} s^{q-1}+\cdots \cdots d_{1} s+d_{0}}
$$

Because there are $2 q$ unknowns in the reduced-order system, it is forced to correspond to the first $2 q$ terms of eq.(6) by using Padé approximation, yielding the following equality:

$$
\begin{gathered}
\frac{n_{q-1} s^{q-1}+n_{q-2} s^{q-2}+\cdots \cdots+n_{1} s+n_{0}}{s^{q}+d_{q-1} s^{q-1}+\cdots \cdots d_{1} s+d_{0}} \\
=m_{0}+m_{1} s+\cdots \cdots+m_{2 q-1} s^{2 q-1}
\end{gathered}
$$

Multiplying both sides of eq. (7) by the denominator of the left-hand side yields a set of equations that can be solved for $2 q$ coefficients. After finding roots of the denominator of the reduced-order model, eq. (6) can be expressed as a partial fraction expansion form given by

$$
\hat{H}(s)=\sum_{i=1}^{q} \frac{r_{i}}{s-p_{i}}
$$

where $r_{i}$ is a residue of $\hat{H}(s)$ at the pole $p_{i}$. It is then straightforward to obtain the approximated impulse response $\hat{h}(t)$ from $(8)$.

\section{MATHEMATICAL FRAMEWORK}

Transmission line characteristics are in general described by Telegrapher's equations [19]. Consider the transmission line system shown in Fig. 2. Telegrapher's equations for such a structure can be derived by discretizing the line into infinitesimal sections of length and assuming uniform per unit length parameters of resistance $(R)$, inductance $(L)$, conductance $(G)$, and capacitance $(C)$. Each section, as shown in Fig. 3. then includes resistance $R \Delta x$, inductance $L \Delta x$, conductance $G \Delta x$, and capacitance $C \Delta x$. Using Kirchhoff's

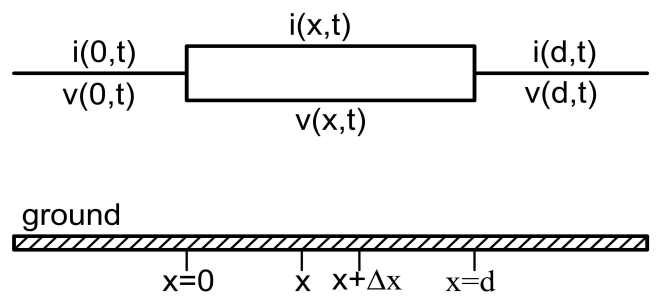

Figure 2: A Typical Transmission Line System

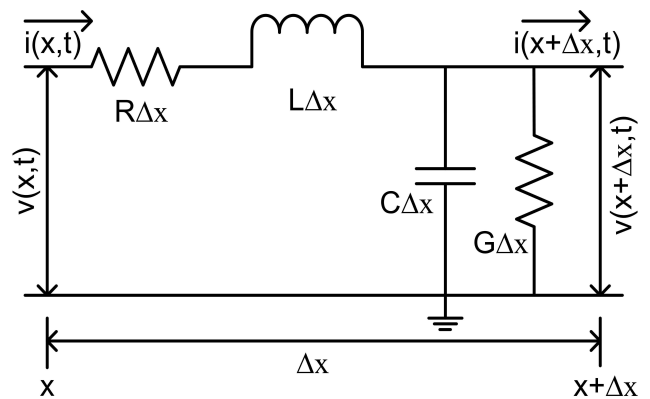

Figure 3: Cross Section of a Transmission Line 
current and voltage laws, one can write [18]

$$
v(x+\Delta x, t)=v(x, t)-R \Delta x i(x, t)-L \Delta x \frac{\partial}{\partial t} i(x, t)
$$

or

$$
\frac{v(x+\Delta x, t)-v(x, t)}{\Delta x}=-R i(x, t)-L \frac{\partial}{\partial t} i(x, t)
$$

Taking the limit $\Delta x \rightarrow 0$, one gets

$$
\frac{\partial}{\partial x} v(x, t)=-R i(x, t)-L \frac{\partial}{\partial t} i(x, t)
$$

Similarly, the second transmission line equation can be obtained as

$$
i(x+\Delta x, t)=i(x, t)-G \Delta x v(x, t)-C \Delta x \frac{\partial}{\partial x} i(x, t)
$$

substituting eq. (9) in (12) we have

$$
\begin{aligned}
& i(x+\Delta x, t) \\
& \quad=i(x, t)-G \Delta x\left(v(x, t)-R \Delta x i(x, t)-L \Delta x \frac{\partial}{\partial t} i(x, t)\right) \\
& \quad-C \Delta x \frac{\partial}{\partial t}\left(v(x, t)-R \Delta x i(x, t)-L \Delta x \frac{\partial}{\partial t} i(x, t)\right)
\end{aligned}
$$

or

$$
\begin{aligned}
& \frac{i(x+\Delta x, t)-i(x, t)}{\Delta x}=-G v(x, t)-C \frac{\partial}{\partial t} v(x, t)+\Delta x \\
& \quad \times\left(G R i(x, t)+(G L+R C) \frac{\partial}{\partial t} i(x, t)+L C \frac{\partial^{2}}{\partial t^{2}} i(x, t)\right)
\end{aligned}
$$

Taking limit $\Delta x \rightarrow 0$, one gets

$$
\frac{\partial}{\partial x} i(x, t)=-G v(x, t)-C \frac{\partial}{\partial t} v(x, t)
$$

Taking the Laplace transform of eq. 11 and eq. 15

$$
\begin{gathered}
\frac{\partial}{\partial x} V(x, s)=-(R+s L) I(x, s)=-Z I(x, s) \\
\frac{\partial}{\partial x} I(x, s)=-(G+s C) V(x, s)=-Y V(x, s)
\end{gathered}
$$

Where $Z$ and $Y$ represent the per unit length impedance and admittance of the transmission line, and given by

$$
Z=R+s L, \quad Y=G+s C
$$

The set of equations represented by (16) and (17) can be solved if they can be written in terms of one of the unknowns $[V(x, s) \operatorname{or} I(x, s)]$ as follows

$$
\begin{gathered}
\frac{\partial^{2}}{\partial x^{2}} V(x, s)=Z Y V(x, s)=\gamma^{2} V(x, s) \\
\frac{\partial^{2}}{\partial x^{2}} I(x, s)=Y Z I(x, s)=\gamma^{2} I(x, s)
\end{gathered}
$$

Where $\gamma(s)$ is the complex propagation constant and it is given by

$$
\gamma(s)=\alpha+j \beta=\sqrt{Z Y}=\sqrt{(R+s L)(G+s C)}
$$

Where $\alpha$ is called the real part of the propagation constant and is known as the attenuation constant, whose units are expressed in nepers $/ \mathrm{m}$. $\beta$ represents the imaginary part of the propagation constant and is known as the phase constant, whose units are expressed in radians $/ \mathrm{m}$.

The generalized dynamic equations of RLC circuit shown in Fig.3 can be written as

$$
M \ddot{V}(x, s)+K V(x, s)=F(x, s)
$$

$$
M \ddot{I}(x, s)+K I(x, s)=G(x, s)
$$

where $K$ and $M$ are governing equations of original model. Observe from eq. (19) and eq. (20) $\frac{K}{M}=-\gamma^{2}$ and $F(x, s)$ and $G(x, s)$ are two different excitations.

\section{SUBSPACE ITERATION SCHEME}

1. Choose set of $(m)$ linearly independent voltages (currents) and construct a subspace $\varphi_{m}^{(0)}$ in which the columns are occupied by voltages (currents).

2. This subspace is considered as an initial approximation of the original order transfer function.

3. for $i=1,2, \ldots \ldots$ the following two steps are applied to solve for the $(i+1)^{t h}$ approximation of poles of the transfer function.

3.1 A set of new subspace $X_{m}^{(i+1)}$ is obtained by the simultaneous inverse iteration. i.e.

$$
K X_{m}^{(i+1)}=M \varphi_{m}^{(i)}
$$

If the iterations proceeded using $X_{m}^{(i+1)}$ as the next estimation of the subspace, the subspace would collapse to the subspace of dimension ' 1 ' and only contain the voltages (currents) corresponding to the a few number of poles. Therefore, RayleighRitz procedure is adopted.

3.2 Compute the reduced order matrices $V \& I$ in the subspace spanned by $X_{m}^{(i+1)}$ :

$$
\begin{aligned}
& K_{R}^{(i+1)}=\left(X_{m}^{(i+1)}\right)^{T} K X_{m}^{(i+1)} \\
& M_{R}^{(i+1)}=\left(X_{m}^{(i+1)}\right)^{T} M X_{m}^{(i+1)}
\end{aligned}
$$

where $K_{R}^{(i+1)}$ and $M_{R}^{(i+1)}$ are the governing equations of reduced model.

4. Now generate the dynamic equations of reduced order model using $K_{R}^{(i+1)}$ and $M_{R}^{(i+1)}$.

\section{SIMULATION RESULTS}

A distributed linear RLC circuit as shown in Fig.4. with all its capacitors and inductors initially charged to a unit voltage and unit current were considered for this example. The order of the matrix was $250 \times 250$.

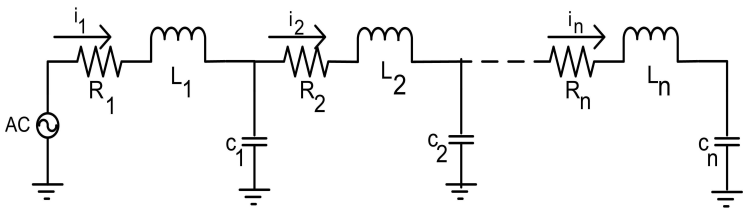

Figure 4: A Typical RLC Circuit

The proposed subspace iterative method, has been used to find the order reduction of the system by considering the derivatives of the linear network. The response of the network is calculated using the proposed scheme. This result is shown in Fig. 5. 


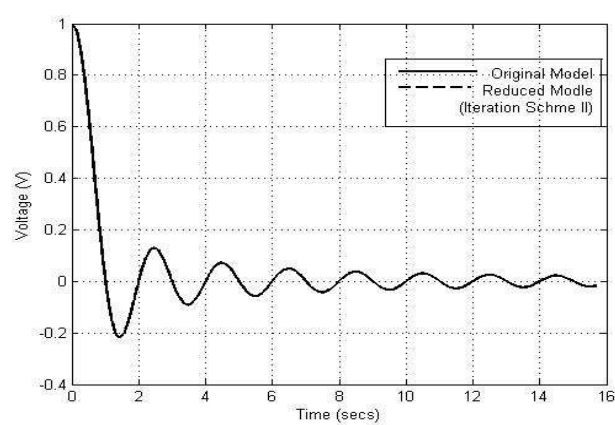

Figure 5: Response of the Reduced Model using Subspace Iterative Scheme

\subsection{Time and Frequency Responses}

As a sample of results, for the Fig. 6, comparison with the output across one of the nodes using subspace iterative method, obtained from the original model and reduced model is shown in Fig. 6. and as seen both the responses match accurately.

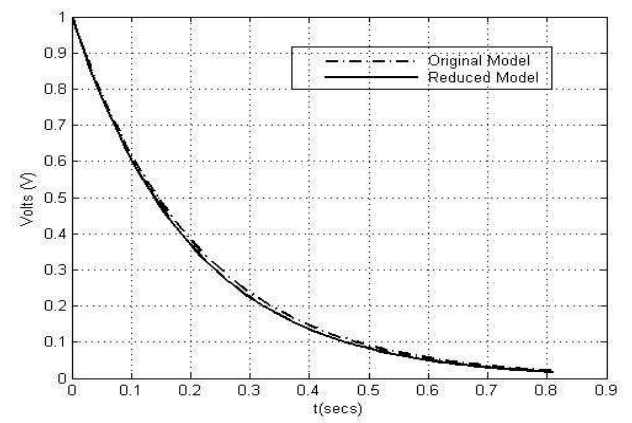

Figure 6: Transient Response Comparison

Similarly, the Bode plots are shown for both original and reduced model in Fig. 7. which clearly indicate that the proposed subspace iterative scheme gives better frequency response matching at all frequencies of interest while the stability of the original system preserved.

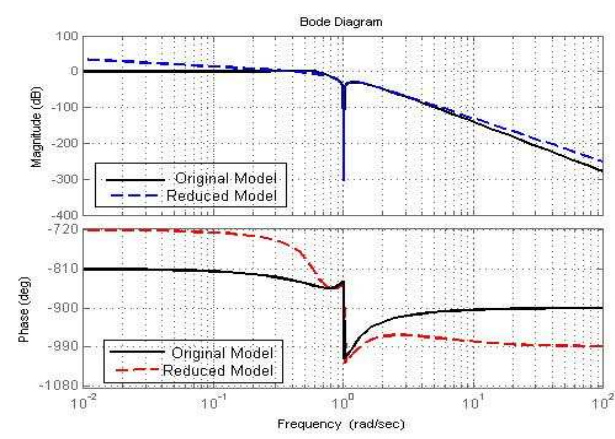

Figure 7: Frequency Response Comparison

\subsection{Comparison with Existing Techniques}

To demonstrate the efficiency achievable by using the subspace iterative scheme, we use the RLC ladder circuit in Fig. 4 again and reduce a sequence of models with sizes 10, 50, 250, and 1000. For each model, the reduced model governing equations have been obtained using proposed algorithm. All these results are tabulated in Table 1. The proposed

Table 1: Reduced Model Order using Proposed Scheme

\begin{tabular}{|c||c||c||c||c|}
\hline Full Modle Size & 10 & 50 & 250 & 1000 \\
\hline Subspace Iterative Scheme & 5 & 19 & 116 & 87 \\
\hline
\end{tabular}

technique, subspace iterative scheme is compared with the existing techniques and the results are tabulated in Table 2.

Table 2: Comparison with Existing Techniques

\begin{tabular}{|c||c||c|}
\hline Method & Order (1000) & Efficiency \% \\
\hline Dian [3] & 182 & 82 \\
\hline Mike [4] & 347 & 65 \\
\hline Telsen [5] & 123 & 88 \\
\hline Proposed Scheme & 87 & 92 \\
\hline
\end{tabular}

\section{CONCLUSIONS}

A novel approach has been presented to create a reducedorder model based on the model reduction technique. To handle the very large matrices in nanometer designs, the original linear system equations describing a distributed network should be replaced by small and dense matrices which form a reduced-order model to reduce the complexity and CPU time of the system. This technique reduces the size of the original model by the $92 \%$, representing an efficient way of handling complex VLSI systems and much suitable for nanometer designs.

\section{REFERENCES}

[1] S. Kumashiro, R. Rohrer, and A. Strojwas, "A new efficient method for the transient simulation of 3-D interconnect structures,"In Proceedings of International Electron Devices Meeting, Dec. 1990, pp. 193-196.

[2] H. W. Jhonson and M. Grahaml, Reading "High-Speed Digital Design" Englewood Cliffs, NJ: Prentice-Hall, 1993.

[3] Dian Zhou, Wei Li, Wei Cai, Nailong Guo, "An Efficient Balanced Truncation Realization Algorithm for Interconnect Model Order Reduction" In proceedings of International Symposium on Circuits and Systems (ISCAS), 2001, Vol 5, pp. 383-386.

[4] Mike Chou, Jacob K. White, "Efficient Formulation and Model-Order Reduction for the Transient Simulation of Three-Dimensional VLSI Interconnect" In Proceedings of IEEE Trans.CAD, Vol. 16, No. 12,December 1997, pp. 1454-1476.

[5] M Telescu, N. Tanguy, P. Brehonnet, P. Vilbe, L.C. Calvez "Low Complexity Equivalent Circuit Models for VLSI Interconnects" In proceedings of 10th IEEE workshop on Signal Propagation on Interconnects (SPI), May 2006, pp 271-273. 
[6] Eli Chiprout and Michel S . Nakhla, "Analysis of Interconnect Networks Using Complex Frequency Hopping (CFH)" In Proceedings of IEEE Trans. CAD, Vol. 14, No. 2, February 1995, pp. 186-200.

[7] Stefano Grivet-Talocia, Andrea Ubolli "On the Generation of Large Passive Macromodels for Complex Interconnect Structures"In Proceedings of IEEE Transactions On Advanced Packaging, Vol. 29, No. 1, February 2006, pp. 39-54.

[8] Ramachandra Achar, Michel S. Nakhla, Qi-Jun Zhang, "Full-Wave Analysis of High-Speed Interconnects Using Complex Frequency Hopping"In Proceedings of IEEE Trans.CAD, Vol. 17, No. 10, October, 1998, pp. 997-1016.

[9] Pavan K. Gunupudi, Michel Nakhla, Ramachandra Achar "Simulation Of High-Speed Distributed Interconnects Using Krylov-Space Techniques" In Proceedings of IEEE Trans.CAD, Vol. 19, No. 7, July 2000, pp. 799-808.

[10] Pavan K. Gunupudi, Michel Nakhla, "ModelReduction of Nonlinear Circuits using Krylov Space Techniques"In Proceedings of IEEE/ACM Design Automation Conference (DAC), New Orleans, Louisiana, June 2001, pp. 13-16.

[11] J. B. Faria, Reading "Multiconductor Transmission Line Structures". New York: Wiley, 1993.

[12] C Jiao, A. C. Cangellaris, A. Yaghmour, J. L. Prince, "Sensitivity analysis of multiconductor transmission lines and optimization for highspeed interconnect design," In Proceedings of IEEE Transactions on Comp., Pack., Manuf. Tech, pp. 132141, May 2000.
[13] I.Wuyts, D. De Zutter, "Circuit model for plane-wave incidence on multiconductor transmission lines," In Proceedings of IEEE Transactions on Electromagnatics Compatibility, vol. 36, pp. 206212, Aug. 1994.

[14] L. Daniel, C. S. Ong, S. C. Low, K. H. Lee, J. White, "Geometrically parameterized interconnect performance models for interconnect synthesis" In Proceedings of International Symposium on Physical Design, San Diego, CA, 2002, pp. 202-207.

[15] Y Liu, L. Pileggi, A. Strojwas, "Model order-reduction of $\mathrm{RC}(\mathrm{L})$ interconnect including variational analysis," In Proceedings of $36^{\text {th }}$ Design Automation Conference, New Orleans, LA, 1999, pp. 201-206.

[16] G. Shi, C.-J. Shi, "Parametric reduced order modeling for interconnect analysis," In Proceedings of ASPDAC, Yokohama, Japan, 2004, pp. 774-779.

[17] Payam Heydari, Massoud Pedram, "Model-Order Reduction Using Variational Balanced Truncation With Spectral Shaping" In Proceedings of IEEE Trans.CASCircuits And Systems-I: Regular Papers, Vol. 53, No. 4, April 2006.

[18] Zu-Qing Qu, Reading "Model order Reduction Techniques with Applications in Finite Element Analysis" Springer Publications 2004, ISBN: 1852338075.

[19] C. Paul, Reading "Analysis of Multiconductor Transmission Lines". New York: Wiley, 1994. 